\title{
Referenda and the Provision of a Binary Public Good
}

\author{
Rajat Deb', Indranil K. Ghosh ${ }^{2}$, Tae Kun Seo ${ }^{1}$ \\ ${ }^{1}$ Department of Economics, Southern Methodist University, Dallas, USA \\ ${ }^{2}$ Graham School of Management, Saint Xavier University, Chicago, USA \\ Email:rdeb@mail.smu.edu, ghosh@sxu.edu, tseo@mail.smu.edu
}

How to cite this paper: Deb, R., Ghosh, I.K. and Seo, T.K. (2018) Referenda and the Provision of a Binary Public Good. Journal of Mathematical Finance, 8, 668-689. https://doi.org/10.4236/jmf.2018.84042

Received: August 23, 2018

Accepted: November 23, 2018

Published: November 26, 2018

Copyright (c) 2018 by authors and Scientific Research Publishing Inc. This work is licensed under the Creative Commons Attribution International License (CC BY 4.0).

http://creativecommons.org/licenses/by/4.0/

\section{c) (i) Open Access}

\begin{abstract}
In referenda, projects are approved if the support for the project exceeds a particular threshold or quota. The usual threshold is the requirement of majority support. The paper provides a theoretical analysis of referenda with different threshold quotas for the provision of a binary public good. Optimal quota sizes are characterized and how the optimal quota changes as the size of the society increases is studied. While the majority quota may or may not be optimal, the conditions which determine the significance of the loss of expected welfare from the (possibly inoptimal) use of the majority quota are analyzed and interpreted. It is shown that the welfare loss from using an inoptimal majority quota will be insignificant if the ratio of the average intensity of support for the project relative to the intensity of opposition to it is positively associated with the probability of a positive net valuation of the project.
\end{abstract}

\section{Keywords}

Referendum, Optimal Quota, Optimal Proportion, Expected Welfare, Public Good

\section{Introduction}

Whether it is a new public library for a community or whether it is a project to maintain and improve common areas in a condominium complex, projects which provide non-rivalrous benefits to members belonging to some group, invariably involve shared costs and differential benefits. Decisions about such public projects are often based on referenda. For approval of the project, usually, a minimum threshold of support needs to be met. This often means making sure that a majority of the agents support the proposed project. For small groups, the 
requirement for support could be more stringent with even single individuals sometimes being granted the power to veto a proposal. If the project is approved, then the cost of the project is shared. For private groups, such as homeowners' associations, most often, every member pays the same amount. In this paper, we study the problem of optimal public good provision with equal cost sharing using referenda that employ a fixed threshold quota.

Many justifications based on axiomatic characterizations ${ }^{1}$ can be found in the theoretical literature for the use of referenda ${ }^{2,3}$. The characterization theorem in [12], for instance, implies that under standard continuity assumptions any strategy-proof, non-bossy and anonymous cost sharing rule is a quota rule (a referendum) with equal cost sharing: the class of rules considered in this paper. An alternative approach justifies referenda as good approximations to the optimum. For instance in [13] it is argued that “...in large populations for any interim efficient allocation rule, there exists a corresponding referendum that yields approximately the same total welfare. Moreover, if there is a common value component to the voters' preference, then there is an unique approximating referendum". Thus, the use of referenda for public good provision can be justified as a low cost method of approximating a class of optimal rules ${ }^{4}$.

However, the strongest reasons for studying quota based referenda for public good provision arise not from the theoretical mechanism design literature, that we have referred to above, but from the increasing practical importance of these rules in the real world. Most private groups, when considering projects involving the provision of a non-rivalrous good to their members make extensive use of referenda. At the level of the government, Switzerland has long been known for using direct democracy (referenda) for making public decisions. In the USA there has been a significant growth of direct public initiatives at the state and local level as described in [15], [16] and [17] and with it an increased use of "direct democracy" for decisions on public projects. Such decisions relate to sports stadiums, highway construction, public transportation, flood control, parks projects, etc. Two interesting examples are the 2007 referendum on the Trinity River Corridor Project in Dallas (approved) and the Transportation Action Plan (I-5, Puget Sound Project) in Seattle (rejected). In all, there were 544 ballot propositions, including direct public initiatives between 2010 and 2017 in the US [17] .

\footnotetext{
${ }^{1}$ For instance in [1], this threshold is characterized for a budget balanced mechanism satisfying ex-post individually rationality and incentive compatibility.

${ }^{2}$ The theoretical literature studies mechanisms under which honest revelation is an equilibrium strategy. We can distinguish between two broad approaches: 1) The "Strategy-proofness" approach: requiring truth telling be a dominant strategy ([2]-[7]). 2) The "Nash implementability" approach: which has the weaker requirement that honest revelation be a Nash equilibrium. (This literature is vast with [8] and [9] being representative examples). For a recent contribution to the Cost Sharing literature for public goods see [10].

${ }^{3}$ For a justification for using voting mechanisms for the provision of public goods based on robust coalition proofness and incentive compatability see [11].

${ }^{4}$ For arguments analyzing the majority quota see [14].

${ }^{5}$ Of the 146 ballot initiatives in 2014, 59 were transportation related (see [17]).
} 
Setting thresholds for a referendum at different levels has implications for the welfare generated for the group. If the quota of support required for approval of the project is set too high, let us say if unanimous agreement is required, too many desirable projects would be rejected. If the quota is set too low, say for instance if a project is accepted whenever some pre-specified small number of individuals support it, too many undesirable projects would be accepted. Recognizing this trade-off, we focus on two issues:

First, we address the three following questions: 1) What are the determinants of the optimal quota? 2) Can the expected welfare from a prespecified distribution of net valuations be exactly mirrored by a specific (closed form) family of distributions? 3) How does the optimal quota vary with cost of the project?

Second, we analyze the significance of NOT using the optimal quota. We know that if we do not use the optimal quota the welfare will not be maximized; but will this loss of welfare necessarily be large or will it be insignificant? This question is of great practical importance (even more so than the actual calculation of the optimal quota) because in the real world, the majority quota, whether or not this quota is the optimal, is the one most frequently used.

We proceed as follows:

First, we analyze welfare valuations that are independently and identically (but otherwise arbitrarily) distributed and derive a formula for expected welfare and the optimal quota. We then show that a simple three parameter family of distributions-the two part uniform distribution-is such that, for any arbitrary pre-specified distribution $F$ of net valuations, by appropriately selecting the three parameters of the two part uniform distribution, the two part uniform distribution will give exactly the same expected welfare as the pre-specified distribution of net valuations, $F$. Thus, computational results about expected welfare which are true for this specific family of distributions will be valid for all distributions.

We conclude the first part of our analysis by examining how the per capita cost of the project affects the optimal quota. We show that if the probability distribution of net valuations is relatively "fat tailed" (i.e., net valuations are extreme and do not have a high probability of being in the neighborhood of the neutral value of zero) then an increase in the per capita cost increases the optimal quota. If the probability distribution of net valuations is relatively "thin tailed" (i.e., net valuations have a high probability of being in the neighborhood of zero) an increase in the per capita cost will have the opposite effect, decreasing the optimal quota. We conclude this part of the paper by examining effect of population size on the optimal quota before turning to analyzing the consequence of using inoptimal quotas.

To analyze inoptimal quotas, we use the properties of the two part uniform distribution to evaluate the expected welfare loss from use of inoptimal rules. This helps us quantify the impact of the possible inoptimality arising from the 
pervasive use of the majority quota ${ }^{6}$. We argue that when the majority quota is different from the optimal quota, the use of the majority quota may or may not lead to any perceptible ${ }^{7}$ loss of expected welfare. Even when the difference between the majority quota and the optimal quota is large, the majority quota will perform extremely well when the ratio of the positive to the negative mean is large. This can be interpreted as indicating that the majority quota will perform well when there is a stronger average intensity of support for the project among supporters relative to the intensity of opposition to it among the opponents. Analogously, the loss in expected welfare from using the majority quota (when the majority quota is not optimal) will be large when the ratio of the positive to the negative mean is small and thus the use of the majority quota in the presence of intense minority opposition is likely to give unsatisfactory results.

\section{A Motivating Example}

The following example illustrates our problem and the logic of the underlying approach we take to solve it.

Consider a set of three individuals $N=\{1,2,3\}$ who are the owners of three condominiums in a common building having the three units. Decisions about maintenance and improvement projects are made by the three individuals. The public decision making entity is the "homeowners' association" of which all three individuals are members. Let the society be considering a public project (say, the installation of a motion activated security system). The individuals may consider the public project (the motion activated security light) as something beneficial (enhancing security) or as a nuisance (with a propensity for false alarms triggered by cats and other small animals). The project impacts the utility a of all the individuals positively or negatively. Suppose the cost of the project is $\$ 3$. If undertaken each member of the society will have to pay $\$ 1$. Let there be two types, high and low, valuing the consumption of the public good at $\$ 2$ and $-\$ 2$, respectively. The type of an individual is assumed to be private information. Each individual has an equal probability of being one type or the other; the valuation of the public good of one individual being stochastically independent of the valuations of the others. Three approval quotas ( $\alpha$-quotas) are possible for a referendum. The approval of the project can require: 1) unanimous support ( $\alpha=3$ ),2) majority support ( $\alpha=2$ ) or 3) just the support of a single member ( $\alpha=1$ ). Which of these three rules should be adopted?

Under our assumptions, in this example, there are eight possible societies: $\{(2,2,2),(-2,2,2),(2,-2,2),(2,2,-2),(-2,-2,2),(-2,2,-2),(2,-2,-2)$, $(-2,-2,-2)\}$ with each having an equal probability of occurring. Our optimal $\alpha$ for a referendum will be the one that maximizes the expected value of the sum of individual utilities ("expected welfare") over the eight states. Notice that assigning equal probabilities to each of the eight possible states leads to the ${ }^{6}$ For an alternative approach to this problem see [9], [13] and [18].

${ }^{7} \mathrm{We}$ define a perceptible difference to be numerical differences in percentage terms that can be distinguished by computers using 32-bit technology. 
probability of unanimous acceptance (respectively, rejection) of $\frac{1}{8}$. Similarly, the probability of a majority being for (respectively, against) the proposal is $\frac{3}{8}$.

Table 1 shows the expected welfare calculations for the above project. For instance, recalling that the cost of the project is $\$ 3$, if $\alpha=1$ and exactly one individual has a valuation of 2 , the project would be accepted and the welfare generated would be $2-2-2-3=-5$. This is shown by the entry -5 in the third column of Table 1. We can find the expected welfare, based on the example given above, for each of these four cases (unanimous acceptance, unanimous rejection, majority acceptance and majority rejection) and hence find the optimal $\alpha$ (in this case the optimal value of $\alpha$ is 3 ).

For this example the consequences of using the an inoptimal $\alpha$ can be substantial and would result in a loss of expected welfare (as compared to the optimum) of at least $33 \%$ (This is caculated by taking the difference between the percentage change between the optimal expected welfare 0.375 and the next highest expected welfare 0.25 in the table above).

Using the approach described in the example above, we will provide a closed form solution for the general case for a society with $n$ individuals whose valuations are identically and independently but otherwise arbitrarily distributed. Using our formula for the optimum we will examine the implications of changes in factors that determine this optimum, conditions under which the majority quota will be the optimum and the welfare consequences of using the majority quota when it is not the optimal rule.

\section{The Model and Notation}

Let the group of agents be given by $N=\{1,2,3, \cdots, n\}, n \geq 2$. Let $y \in\{1,0\}$ be a binary public good which may be consumed by the members of $N$. Consider the problem of whether to provide the good $(y=1)$ or not $(y=0)$ when the cost of the project $C(n)>0$ is a function of $n$ and when if the good is produced every individual pays the amount $\gamma(n)=\frac{C(n)}{n}$. (If the public good is not produced, then no money is paid by any of the individuals in $N$ ).

Let the (discrete, continuous, or mixed) random variables $X_{i}$ for $i=1,2, \cdots, n$ represent the individuals' valuations of the public good. The $X_{i}$ s are assumed to

Table 1. Expected welfare of the public good.

\begin{tabular}{ccccc}
\hline State & Probability & $\alpha=1$ & $\alpha=2$ & $\alpha=3$ \\
\hline Three $2 \mathrm{~s}$ & $1 / 8$ & 3 & 3 & 3 \\
Two 2s & $3 / 8$ & -1 & -1 & 0 \\
One 2 & $3 / 8$ & -5 & 0 & 0 \\
Zero 2s & $1 / 8$ & 0 & 0 & 0.375 \\
Expected Welfare & & -1.625 & 0.25 &
\end{tabular}


be independently and identically distributed (i.i.d) and to be Stieltjes integrable ${ }^{8}$. Depending on the realizations of their types, different individuals will have (possibly) different valuations of the public good. A realization of $X_{i}^{\text {'s }}$, individual is (gross) valuation of the public good, is denoted by $x_{i}$ (which may be negative). Hence, individual $i$ s net valuation of the public good is $v_{i}(n)=x_{i}-\gamma(n)$. Since $v_{i}(n)$ is the realization of the random variable, $V_{i}(n)=X_{i}-\gamma(n)$ and since for any given $n$, the $X_{i}^{\prime}$ s are Stieltjes integrable and i.i.d., the random variables $V_{i}(n)$ for $i=1,2, \cdots, n$ will also be Stieltjes integrable and i.i.d. Denote the $c d f$ of $V_{i}(n)$ by $F_{i}^{n}=F^{n}$. To avoid triviality, we will assume that both positive and negative net valuations are possible.

Let $\alpha \in\{1,2, \cdots, n\}$ An $\alpha$-quota referendum rule for provision of a public good is given by:

$$
\begin{gathered}
y=1 \text { if } \bar{N}=\left\{i: v_{i}(n) \geq 0\right\} \subseteq N \text { is such that }|\bar{N}| \geq \alpha ; \\
y=0, \text { otherwise. }
\end{gathered}
$$

$\alpha=1$ requires unanimity in that the project is carried out if and only if there is no opposition. The majority quota rule is given by the case where $\alpha=\left\lceil\frac{n}{2}\right\rceil$, where $\left\lceil\frac{n}{2}\right\rceil$ is the smallest integer greater than or equal to $\frac{n}{2}$.

A project in a full information first best environment is a desirable project if the sum of net valuations is greater than or equal to zero. If the sum of net valuations is negative, the project is an undesirable one which, if one had full information, would be rejected. For realization of the random variables for which $|\bar{N}| \geq \alpha$ for desirable projects or where $|\bar{N}|<\alpha$ for undesirable projects, the first best welfare level is attained and only desirable projects are accepted and undesirable ones rejected. However, there will be realizations for which an $\alpha$-quota referendum will make "mistakes", rejecting $(|\bar{N}|<\alpha)$ desirable projects and accepting $(|\bar{N}| \geq \alpha)$ undesirable ones. In these cases a welfare loss will occur. Since for each realization, the full information first best decision (choosing desirable projects and rejecting undesirable ones) gives us the maximum possible welfare for that realization, choosing a quota $\alpha$ for an $\alpha$-quota referendum so as to minimize the expected losses from "mistakes" is equivalent to choosing an $\alpha$ so as to maximize the expected welfare from an $\alpha$-quota referendum.

Let $W(\alpha, n)$ be the random variable denoting the welfare generated by the $\alpha$-quota referendum. Then, the optimal quota (which is a function of $n$ ) is defined as:

$$
\arg \max _{\alpha}[E(W(\alpha, n))] .
$$

We will provide a specific formula for the optimal quota in terms of positive and negative means of the distribution of net valuations defined as follows:

${ }^{8}$ This allows us to deal with continuous, discrete and mixed distributions. 
For our public good, the positive mean of $v_{i}(n), \mu^{+}(n)$ is defined as $\mu^{+}(n)=\frac{\int_{0}^{\infty} t \mathrm{~d} F^{n}(t)}{1-F^{n}(0)}$. This is the (conditional) mean of the positive net valuations given $v_{i}(n) \geq 0$.

Analogously, $\mu^{-}(n)=\frac{\left|\int_{-\infty}^{0} t \mathrm{~d} F^{n}(t)\right|}{F^{n}(0)}$ defines the negative mean of $v_{i}(n)$.

The positive means can be interpreted as measuring the average intensity of the support for the project among the supporters while the negative means give us the average intensity of the opposition to the project among those who oppose the project.

\section{Expected Welfare and Optimal Quota with Fixed $n$}

To derive an expression for expected welfare we first develop some notation to identify the expected welfare generated by some particular subsets of realizations.

For each integer $q, 1 \leq q \leq n$, let $D(q)$ be the set of realizations for which exactly the first $q$ individuals have non-negative valuations:

$$
D(q)=\left\{\left(v_{1}, v_{2}, \cdots, v_{n}\right) \in \mathcal{R}^{n}:\left(\text { i) } 0 \leq v_{1}, v_{2}, \cdots, v_{q} \& \text { (ii) } v_{q+1}, \cdots, v_{n}<0\right\} .\right.
$$

For any integer $q, 1 \leq q \leq n$, the expected welfare from projects in which exactly the first $q$ individuals have non-negative valuations, is given by:

$$
\Gamma(q)=\int \cdots \int_{D(q)} \sum_{i=1}^{n} v_{i} \mathrm{~d} F\left(v_{1}\right) \mathrm{d} F\left(v_{2}\right) \cdots \mathrm{d} F\left(v_{n}\right) .
$$

Noting that there are $\left(\begin{array}{l}n \\ q\end{array}\right)=\frac{n !}{q !(n-q) !}$ possibly distinct ways of selecting the "first $q$ individuals" from the set of $n$ individuals, the expected welfare, $\tilde{\Gamma}(q)$, generated by all realizations with exactly $q$ individuals (though not necessarily the first $q$ ) having non-negative net valuations is given by:

$$
\tilde{\Gamma}(q)=\left(\begin{array}{l}
n \\
q
\end{array}\right) \Gamma(q) .
$$

For any $\alpha \in\{1,2, \cdots, n\}$ if one uses an $\alpha$-quota referendum, for all those realizations of net valuations of the project with $q$ non-negative valuations where $q \geq \alpha$, the project is accepted. Hence, using (3) and summing over all $q$ 's, $\alpha \leq q \leq n$, a precise expression for the expected welfare, $E(W(\alpha, n))$, in terms of the cdfs of the net valuations can be written as:

$$
E(W(\alpha, n))=\sum_{q=\alpha}^{n} \tilde{\Gamma}(q)=\sum_{q=\alpha}^{n}\left(\begin{array}{l}
n \\
q
\end{array}\right) \Gamma(q) .
$$

We will first characterize the optimal quota with a fixed $n$ before examining the implications of variations in $n$. In this section, to lighten our notation, we will drop " $n$ " from our notation", wherever the $n$, because of its constancy, has

${ }^{9}$ Thus, $W(\alpha, n)$ will be reduced to $W(\alpha)$. 
no impact on the analysis or where the impact is obvious.

The key to finding the expected welfare maximizing $\alpha$ is to focus on $\Delta(\alpha)$, the change in the expected welfare as $\alpha$ changes from $\alpha$ to $\alpha+1$.

Using (4), this is given by:

$$
\Delta(\alpha)=E(W(\alpha+1))-E(W(\alpha))=-\left(\begin{array}{l}
n \\
\alpha
\end{array}\right) \Gamma(\alpha) .
$$

From (4) and (5), we can see that both the problem of calculating the expected welfare from an $\alpha$-quota rule and that of measuring the impact on expected welfare of an unit increase in $\alpha$, can be reduced to computing the value of the integral $\Gamma(\alpha)$.

Using (2), we can proceed as follows:

$$
\begin{aligned}
\Gamma(\alpha)= & \int \cdots \int_{D(\alpha)} \sum_{i=1}^{n} v_{i} \mathrm{~d} F\left(v_{1}\right) \mathrm{d} F\left(v_{2}\right) \cdots \mathrm{d} F\left(v_{n}\right) \\
= & \int \cdots \int_{D(\alpha)} \sum_{i=1}^{\alpha} v_{i} \mathrm{~d} F\left(v_{1}\right) \mathrm{d} F\left(v_{2}\right) \cdots \mathrm{d} F\left(v_{n}\right) \\
& +\int \cdots \int_{D(\alpha)} \sum_{i=1+\alpha}^{n} v_{i} \mathrm{~d} F\left(v_{1}\right) \mathrm{d} F\left(v_{2}\right) \cdots \mathrm{d} F\left(v_{n}\right) .
\end{aligned}
$$

Using the definition of $D(q)$ in (1) with $q=\alpha$ we know that $0 \leq v_{1}, v_{2}, \cdots, v_{\alpha}<\infty$ and $-\infty<v_{\alpha+1}, \cdots, v_{n}<0$. Thus, the first term of the sum in (6), $\int \cdots \int_{D(\alpha)} \sum_{i=1}^{\alpha} v_{i} \mathrm{~d} F\left(v_{1}\right) \mathrm{d} F\left(v_{2}\right) \cdots \mathrm{d} F\left(v_{n}\right)$, can be written as:

$$
F(0)^{n-\alpha} \int_{0}^{\infty} \mathrm{d} F\left(v_{\alpha}\right) \int_{0}^{\infty} \mathrm{d} F\left(v_{\alpha-1}\right) \cdots \int_{0}^{\infty} \mathrm{d} F\left(v_{2}\right) \int_{0}^{\infty} \sum_{i=1}^{\alpha} v_{i} \mathrm{~d} F\left(v_{1}\right) .
$$

This simplifies to:

$$
\alpha\left[\int_{0}^{\infty} v_{1} \mathrm{~d} F_{1}\left(v_{1}\right)\right](1-F(0))^{\alpha-1} F(0)^{n-\alpha}=\alpha \mu^{+}(1-F(0))^{\alpha} F(0)^{n-\alpha} .
$$

Similarly, the second term in (6) can be written as:

$$
-(n-\alpha) \mu^{-}(1-F(0))^{\alpha} F(0)^{n-\alpha} .
$$

Thus, (6) reduces to:

$$
\Gamma(\alpha)=\left[\alpha \mu^{+}-(n-\alpha) \mu^{-}\right](1-F(0))^{\alpha} F(0)^{n-\alpha} .
$$

Substituting (7) in (5) gives us:

$$
\Delta(\alpha)=-\left[\alpha \mu^{+}-(n-\alpha) \mu^{-}\right]\left(\begin{array}{l}
n \\
\alpha
\end{array}\right)(1-F(0))^{\alpha} F(0)^{n-\alpha} .
$$

Hence, (4) can be written as:

$$
\begin{gathered}
E(W(\alpha))=-\sum_{q=\alpha}^{n} \Delta(\alpha) \\
=\sum_{q=\alpha}^{n}\left[q \mu^{+}-(n-q) \mu^{-}\right]\left(\begin{array}{l}
n \\
q
\end{array}\right)(1-F(0))^{q} F(0)^{n-q} .
\end{gathered}
$$

We can see from (8), that since $\left(\begin{array}{l}n \\ \alpha\end{array}\right)(1-F(0))^{\alpha} F(0)^{n-\alpha}>0$, we will have: 


$$
\Delta(\alpha) \gtreqless 0 \text { according as } \alpha \lesseqgtr \frac{n \mu^{-}}{\mu^{+}+\mu^{-}} .
$$

To see the economic intuition underlying (11), say $\alpha$ is less than $\frac{n \mu^{-}}{\mu^{+}+\mu^{-}}$ Observe that when $\alpha$ increases to $\alpha+1$ all the realizations with exactly $\alpha$ positive valuations get rejected under the quota $\alpha+1$ and that these realizations were not rejected under the quota $\alpha$. There are no other differences in the evaluation of the different realizations under the two quotas. Moreover, $\left(\begin{array}{l}n \\ \alpha\end{array}\right) \Gamma(\alpha)$ is precisely the expected value of the welfare from these rejected realizations with exactly $\alpha$ positive valuations. From (7), we see that that for all integers $\alpha$ less than $\frac{n \mu^{-}}{\mu^{+}+\mu^{-}},\left(\begin{array}{l}n \\ \alpha\end{array}\right) \Gamma(\alpha)$ (the expected welfare from realizations with exactly $\alpha$ non-negative net valuations) is negative. Now, since an increase of the required quota from $\alpha$ to $\alpha+1$ results in precisely these projects being rejected, such a change will increase the expected welfare. An analogous argument holds for the case when $\alpha$ is greater than $\frac{n \mu^{-}}{\mu^{+}+\mu^{-}}$and $\left(\begin{array}{l}n \\ \alpha\end{array}\right) \Gamma(\alpha)$ is positive and an increase from $\alpha$ to $\alpha+1$ reduces welfare.

Now, for any given probability distribution of net valuations, two cases are possible: 1) $\frac{n \mu^{-}}{\mu^{+}+\mu^{-}}$is an integer. 2) $\frac{n \mu^{-}}{\mu^{+}+\mu^{-}}$is not an integer.

If (1) is true, using (11), for all integers $\alpha$ less than $\frac{n \mu^{-}}{\mu^{+}+\mu^{-}}$an increase from $\alpha$ to $\alpha+1$ will increase expected welfare; for $\alpha=\frac{n \mu^{-}}{\mu^{+}+\mu^{-}}$an increase in $\alpha$ from $\alpha$ to $\alpha+1$, will leave the expected welfare unchanged; and, for $\alpha$ greater than $\frac{n \mu^{-}}{\mu^{+}+\mu^{-}}$an increase from $\alpha$ to $\alpha+1$ will decrease expected welfare. Thus, both $\frac{n \mu^{-}}{\mu^{+}+\mu^{-}}$and $\frac{n \mu^{-}}{\mu^{+}+\mu^{-}}+1$ give us optimal values of $\alpha$.

If (2) is true, then for all integers $\alpha$ less than the smallest integer greater than $\frac{n \mu^{-}}{\mu^{+}+\mu^{-}}, \alpha$ will satisfy $\alpha<\frac{n \mu^{-}}{\mu^{+}+\mu^{-}}$and using (11), this implies that an increase of $\alpha$ from $\alpha$ to $\alpha+1$ will increase expected welfare. Similarly, for all integers $\alpha$ greater than the smallest integer greater than $\frac{n \mu^{-}}{\mu^{+}+\mu^{-}}, \alpha$ will satisfy $\alpha>\frac{n \mu^{-}}{\mu^{+}+\mu^{-}}$and using (11), this implies that an increase in $\alpha$ from $\alpha$ to $\alpha+1$ will decrease the expected welfare. Thus, the smallest integer greater than $\frac{n \mu^{-}}{\mu^{+}+\mu^{-}}$gives us the unique optimal value of $\alpha$. Moreover, for smaller 
(respectively, larger) values than the optimal, the welfare will be increasing (respectively, decreasing) in $\alpha$.

Thus, we have shown that in both cases (1) and (2), the smallest integer greater than or equal to $\frac{n \mu^{-}}{\mu^{+}+\mu^{-}}$gives us an optimal value of $\alpha$ and the expected welfare is a (weakly) single peaked function of $\alpha$. It should also be clear that (2) above is the generic case in the sense that in the space of all possible values of $\mu^{+}$and $\mu^{-}$, the points at which (1) holds are isolated points (with Lebesgue measure zero) and for any perturbation, however small, the model will revert to case (2). In this case, the expected welfare is a strictly single peaked function of $\alpha$.

The results derived above are summarized as Proposition 1 below:

Proposition 1. For the public good, the expected welfare for any $\alpha$ is given by the single peaked function:

$$
E(W(\alpha))=\sum_{q=\alpha}^{n}\left[q \mu^{+}-(n-q) \mu^{-}\right]\left(\begin{array}{l}
n \\
q
\end{array}\right)(1-F(0))^{q} F(0)^{n-q}
$$

and the change in expected welfare $\Delta(\alpha)$ can be written as:

$$
\Delta(\alpha)=-\left[\alpha \mu^{+}-(n-\alpha) \mu^{-}\right]\left(\begin{array}{l}
n \\
\alpha
\end{array}\right)(1-F(0))^{\alpha} F(0)^{n-\alpha} .
$$

Since,

$$
\Delta(\alpha) \gtreqless 0 \text { according as } \alpha \lesseqgtr \frac{n \mu^{-}}{\mu^{+}+\mu^{-}},
$$

an optimal quota, which is almost always unique, is given by

$$
\left\lceil\frac{n \mu^{-}}{\mu^{+}+\mu^{-}}\right\rceil \text {. }
$$

Remark 1. Proposition 1 shows that the size of the society, the information about the average intensity of positive support among supporters $\left(\mu^{+}\right)$and the average intensity of the opposition among the opponents $\left(\mu^{-}\right)$together with the probability of a net valuation being negative $(F(0))$ completely determine the expected welfare from the project under any $\alpha$-quota referendum.

Remark 2. The formula of the optimal quota can be derived as a special case of a more general result studying efficiency of voting blocks with different weights contained in [19].

Remark 3. The optimal quota will coincide with the majority quota if and only if the values of the positive and negative means are not too far apart. The majority quota based referendum will underprovide or overprovide the public good according as whether the positive mean is sufficiently greater than or less than the negative mean. The necessary imprecision from the use of the words "too far" and "sufficiently" in the previous two statements is caused by the "rounding up to the higher integer" involved in the characterization of the 
optimal $^{10}$.

Remark 4. If $\frac{\mu^{+}}{\mu^{-}}$is small (i.e., if the relative intensity of feelings against the project is strong among those who oppose the project as compared to the intensity of feelings for it among supporters of the project) then in small groups, the requirement of unanimous consent for proceeding with the project is optimal. (For example, the optimal $\alpha=n=4$, if $\mu^{+}=2, \mu^{-}=8$.)

Remark 5. The optimal value of $\alpha$, is non-decreasing in the negative mean and non-increasing in the positive mean of the net valuations and is independent of any other aspect of the distribution ${ }^{11}$. This has the invariance implication that tells us that it is possible for "large" changes in density to significantly alter the "shape" of the distribution of net valuations and yet for such changes to have no impact on the optimal quota.

In the example below we provide a simple three parameter family of distributions which is such that with the appropriate selection of the three parameters we generate exactly the same expected welfare as that which would be given by any pre-specified distribution of net valuations. Moreover, this family of distributions is such that it is possible to shift arbitrary amounts of probability density from negative to positive valuations or vice versa while leaving $\frac{n \mu^{-}}{\mu^{+}+\mu^{-}}$unaltered. This distribution will allow us to compute expected welfare of inoptimal quota rules to evaluate conditions under which deviations from the optimal quota result in a significant (respectively, an insignificant) loss of welfare.

Example 1. (The Two Stage Uniform Distribution) Let the distribution of net valuations for the public good have supports $-a$ and $b$ where $a, b>0{ }^{12}$. For some arbitrary $\theta, 1>\theta>0$ let the pdf $f$ of net valuations be defined as follows:

$$
f(t)= \begin{cases}\frac{\theta}{a} & \text { if }-a \leq t<0, \\ \frac{1-\theta}{b} & \text { if } 0 \leq t \leq b, \\ 0, & \text { elsewhere }\end{cases}
$$

Here, the negative net valuations have an uniform probability density of $\theta$ and the positive net valuations have an uniform probability density of $(1-\theta)$. Thus, $\mu^{+}=\frac{b}{2}$ and $\mu^{-}=\frac{a}{2}$. Hence, $\frac{n \mu^{-}}{\mu^{+}+\mu^{-}}=\frac{n a}{a+b}$ is independent of $\theta$. In ${ }^{10}$ We show later that as $n$ becomes arbitrarily large the positive and negative means have to be exacly
equal for the optimal quota as a fraction of the population to be given by the majority rule.
${ }^{11}$ Note that, because a rounding up to the next integer is involved, that small perturbations to the
distribution of net valuations may have no impact on the optimal quota even when such a change
affects $\frac{n \mu^{-}}{\mu^{+}+\mu^{-}}$.
${ }^{12}$ The supports - $a$ and $b$ depend on the per capita cost $\gamma=\frac{C(n)}{n}$. However, in this section, $n$ is con-
stant. This ensures that $a$ an $b$ are constant. 
this case Proposition 1 tells us that the optimal quota is completely independent of the movements in density between positive and negative net valuations that can occur because of changes in $\theta^{13}$.

Now, consider any arbitrary pre-specified distribution of net valuations, $F$. Note that for the two part uniform distribution, by selecting $a$ and $b$ and $\theta$ to be equal to twice the negative mean of $F$, twice the positive mean of $F$ and equal to $F(0)$, respectively, the two part uniform for this choice of the parameters will give exactly the same expected welfare as the pre-specified distribution of net valuations, $F$.

\section{Optimal Quotas, Optimal Proportions and Variations in $\gamma$ and $n$}

Let $\tilde{\alpha}(n)=\left\lceil\frac{n \mu^{-}}{\mu^{+}+\mu^{-}}\right\rceil$be the optimal quota for the public good and let $\tilde{\tau}=\frac{\tilde{\alpha}(n)}{n}$ be the corresponding optimal proportion. What happens to these optima as $n$ changes?

We have assumed that the cost of the project $C(n)$ depends on $n$. It is reasonable to assume that it is a non-decreasing function in $n$. An increase in $n$ will generally have two effects on the optimal quota. Firstly, because $n$ occurs in the numerator of the formula being "rounded up" to determine the optimal quota, it will have the direct effect of exerting an upward pressure on the optimal value of the quota. Thus, other things remaining the same, smaller populations should have (weakly) smaller optimal quotas. However, since an increase in $n$ may increase the cost of the project, this increase may have an indirect impact on the optimal quota by changing the positive and negative means of the net valuations by changing the per capita cost $\frac{C(n)}{n}=\gamma$. To isolate this indirect effect, we will first proceed by treating $\gamma$ as if it were autonomous parameter and consider the effect on the positive and negative means of an autonomous change in the per capita cost.

\subsection{Optimal Quotas and Variations in Per Capita Cost}

We will start by making the assumption that the random variables, $X_{i}$, describing gross valuations, $x_{i}$, have $c d f s$ given by $\tilde{F}$ which have well defined $p d f s, \tilde{f}$.

Now, letting $x=t+\gamma$, where $\gamma$ is the per capita cost, the positive mean (of net valuations) $\mu^{+}=\frac{\int_{0}^{\infty} t \mathrm{~d} F(t)}{1-F(0)}$ can be written as

$\frac{\int_{\gamma}^{\infty}(x-\gamma) \mathrm{d} F(x-\gamma)}{1-F(0)}=\frac{\int_{\gamma}^{\infty}(x-\gamma) \mathrm{d} \tilde{F}(x)}{1-\tilde{F}(\gamma)}$. This gives us:

\footnotetext{
${ }^{13}$ The expected welfare at the optimum will, of course, be different depending on the choice of $\theta$.
} 


$$
\mu^{+}=\frac{\int_{\gamma}^{\infty} x \mathrm{~d} \tilde{F}(x)}{1-\tilde{F}(\gamma)}-\gamma .
$$

Observing that $F(0)=\tilde{F}(\gamma), f(0)=\tilde{f}(\gamma)$ and differentiating with respect to $\gamma$ we get:

$$
\frac{\mathrm{d} \mu^{+}}{\mathrm{d} \gamma}=\frac{f(0) \mu^{+}}{1-F(0)}-1
$$

Hence,

$$
\frac{\mathrm{d} \mu^{+}}{\mathrm{d} \gamma} \leq 0 \text { if and only if } \mu^{+} \leq \frac{1-F(0)}{f(0)} .
$$

From (14) it is clear that how $\mu^{+}$changes as $\gamma$ increases depends on whether net valuations have a relatively high probability of being concentrated around zero.

We will say that the distribution describing the net valuations has a relatively fat right tail if it is sparsely distributed at the origin relative to its right hand tail so that $f(0)$ is small enough for $\mu^{+} \leq \frac{1-F(0)}{f(0)}$. Clearly in this case, $\frac{\mathrm{d} \mu^{+}}{\mathrm{d} \gamma} \leq 0$.

Similarly, if the probability distribution describing the net valuations has a relatively thin right tail if the net valuations are relatively more concentrated at the origin and $\mu^{+} \geq \frac{1-F(0)}{f(0)}$. In this case exactly the opposite will be true and $\frac{\mathrm{d} \mu^{+}}{\mathrm{d} \gamma} \geq 0$.

Similarly, we get that

$$
\frac{\mathrm{d} \mu^{-}}{\mathrm{d} \gamma} \geq 0 \text { if and only if } \mu^{-} \leq \frac{F(0)}{f(0)} .
$$

where the comparison of the magnitudes of $\mu^{-}$to $\frac{F(0)}{f(0)}$ tells us about the relative thinness/thickness of the left tail of the distribution of net valuations.

This gives us the following result:

Proposition 2. 1) $\frac{\mathrm{d} \mu^{+}}{\mathrm{d} \gamma} \leq 0$ if and only if $\mu^{+} \leq \frac{1-F(0)}{f(0)}$.2) $\frac{\mathrm{d} \mu^{-}}{\mathrm{d} \gamma} \geq 0$ if and only if $\mu^{-} \leq \frac{F(0)}{f(0)}$.

\subsection{Optimality, Cost Structure and Changes in Population: Some Asymptotic Results}

We can now examine the impact of increasing $n$ on the optimal quota $\tilde{\alpha}$ and the optimal proportion $\tilde{\tau}$.

The overall effect of an increase in $n$ on the optimal quotas $\tilde{\alpha}$ will depend on the cost structure underlying the production of the public good. For any change in $n$, there are three possibilities: 1 ) The cost grows at a rate that is 
greater than $n$ and the per capita cost increases. 2) The cost grows at the same rate as $n$ and the per capita cost remains unchanged. 3) The cost grows at a rate that is slower than $n$ and the per capita cost declines. Given the public ("non-rivalrous") nature of the good, it should be possible to service additional individuals at a negligible additional cost and therefore cases like (1) are highly unlikely and cases like (3) are more likely than cases like (2). With this in mind, we will make the following assumption, one that is satisfied by a number of plausible cost structures: ${ }^{14}$

$$
\frac{\mathrm{d} \gamma}{\mathrm{d} n} \leq 0 \text { and } \lim _{n \rightarrow \infty} \gamma(n)=\gamma_{0} \geq 0 .{ }^{15}
$$

Given assumption (16), using Proposition 2 we get the following result showing that for distributions of net valuations that are relatively thin tailed, (i.e., $\mu^{+} \geq \frac{1-F(0)}{f(0)}$ and $\mu^{-} \geq \frac{F(0)}{f(0)}$ ), the direct impact of increases in $n$ on the optimal quota and the indirect effect via the changes to per capita cost will work in the same direction and the optimal quota will increase as $n$ increases: ${ }^{16}$

Proposition 3. If $\mu^{+} \geq \frac{1-F(0)}{f(0)}$ and $\mu^{-} \geq \frac{F(0)}{f(0)}$ then $\frac{\mathrm{d} \tilde{\alpha}}{\mathrm{d} n} \geq 0$.

As $n$ becomes arbitrarily large and if the optimal quota also increases, it is of some interest to examine if, in the limit, the optimal quota increases at the same rate as $n$, at a faster or a slower rate. In other words, what can we say about the asymptotic optimal proportions: $\tilde{\tau}_{0}=\lim _{n \rightarrow \infty} \tilde{\tau}(n)$ ?

Given our assumption (16), $\gamma_{0}$ is well defined. The asymptotic positive and negative means $\mu_{0}^{+}$and $\mu_{0}^{-}$can be derived from the asymptotic distributions ${ }^{14} \mathrm{~A}$ number of special cases of reasonable cost functions will satisfy this assumption: 1) $C(n)$ is a constant with $C(n)=C_{0} \quad$ (an absolute constant). This could correspond to a public project involving a onetime expenditure with no marginal variation with $n$; for instance erecting a barrier or laying mines along the border to increase national security. In this case, $\lim _{n \rightarrow \infty} \frac{C(n)}{n}=\gamma_{0}=0.2$ ) $C(n)$ is increasing but the cost is absolutely bounded and hence $\lim _{n \rightarrow \infty} \frac{C(n)}{n}=\gamma_{0}=0$ or $C(n)$ grows slowly relative to $n$ and hence, once again, $\lim _{n \rightarrow \infty} \frac{C(n)}{n}=\gamma_{0}=0$. This case is the most likely of the cases and would correspond to such projects as those for supplying drinking water or for supplying sanitation facilities to a community. 3) $C(n)$ grows at the same rate as $n$ and

$\lim _{n \rightarrow \infty} \frac{C(n)}{n}=\gamma_{0}>0$. For instance, $C(n)=K_{0}+\gamma_{0} n, K_{0} \geq 0$ and $\gamma_{0}>0$. Because of the "publicness" aspect of the good we are modeling, this will be a less frequent occurrence than the other cases described above. However, one can imagine scenarios under which this type of cost structure could prevail. This would occur where after a large initial fixed cost, the cost of the project varies proportionally with $n$. For instance, this could correspond to the case where a satellite for monitoring the weather is launched but to receive weather forecasts each agent needs a receiver.

${ }^{15}$ If the per capita cost declines at a faster rate than $\mathrm{n}$ then as $\mathrm{n}$ becomes arbitrarily large the limiting value $\gamma_{0}$ of $\gamma$ would be 0 .

${ }^{16}$ For relatively "thick tailed" distribution of gross valuations the direct and the indirect effect (via changes in $\gamma$ ) of an increase in $n$ work in opposite directions and resulting in an ambiguous impact on the optimal auota. 
of the net valuations where the net valuations are calculated using $\gamma_{0}$ as the price paid by each individual ${ }^{17}$. This gives the asymptotic optimal winning proportion $\frac{\mu_{0}^{-}}{\mu_{0}^{+}+\mu_{0}^{-}}$.

Proposition 4. For the public good if $\gamma_{0} \geq 0$ and $\mu_{0}^{-}>0$ then as $n \rightarrow \infty$ and the optimal quota grows (in the limit) at the same rate as $n$ and the optimal asymptotic proportion is given by $\tilde{\tau}_{0}=\frac{\mu_{0}^{-}}{\mu_{0}^{+}+\mu_{0}^{-}}>0$.

Remark 6. It follows from Proposition 4 that for arbitrarily large $n$, a quota rule based on the majority proportion is optimal if and only if the negative and positive (asymptotic) means are exactly equal. If this is not the case and the positive (respectively, negative) mean is larger, there will be underprovision (respectively, overprovision) of the public good if the majority proportion based referendum is used.

\section{Inoptimality, the Majority Quota and Welfare Loss}

The consequences of deviating from the optimal $\alpha$ will be shown to depend on certain properties of the distribution of net valuations. Since the majority quota is the quota used most often in referenda, in this section we will illustrate the consequences of using the majority quota in referenda for which it is not the optimal. Understanding this case will give us further insights that will enable us to draw more general conclusions with respect to welfare consequences of using inoptimal quotas.

In the following example we show that under certain circumstances large losses may not arise from using the majority quota even when the optimal quota is substantially different from the inoptimal majority quota being used in the sense that there may be no "measurable"18 difference, in percentage terms, between the expected welfare from the optimal quota and the expected welfare generated by the inoptimal majority quota.

Example 2. For this illustration we will use the simple uniform distribution, with the net valuations of the public good being uniformly distributed between $(-a, b)$ for $a, b>0$. From equations (9) and (11) and Proposition 1, $E(W(\alpha))$ is given by

$$
\sum_{q=\alpha}^{n}\left[q \frac{b}{2}-(n-q) \frac{a}{2}\right]\left(\begin{array}{l}
n \\
q
\end{array}\right)\left(\frac{b}{a+b}\right)^{q}\left(\frac{a}{a+b}\right)^{n-q}
$$

and the optimal quota is $\left\lceil\frac{n a}{a+b}\right\rceil$. To avoid duplicating our argument we will focus on $\tilde{\alpha}=\left\lceil\frac{a n}{a+b}\right\rceil<0.5 n$, the case where the majority quota underprovides the public good. Relying on symmetry, these arguments will extend to the case of

${ }^{17} \mathrm{We}$ assume that the analogue of our assumption $0<F_{i}(0)<1$ is satisfied for the asymptotic distributions.

${ }^{18}$ We will clarify this term later. 
overprovision of the good under the majority quota (i.e., where $\tilde{\alpha}=\left\lceil\frac{a n}{a+b}\right\rceil>0.5 n$ ).

Furthermore, since we will primarily be interested in the ratio of the loss of expected welfare from the use of an inoptimal quota as compared to the expected welfare at the optimal quota, a ratio of two functions that are both homogeneous of degree 1 in $a$ and $b$, without any loss of generality, we normalize our selection of $a$ and $b$ so as to restrict the positive and negative means to the unit simplex by setting $\frac{a}{2}+\frac{b}{2}=1$.

As $\frac{a}{b}$ is reduced the optimal quota declines. Consider the cases of the uniform distribution of net valuations when $\frac{b}{2}=\mu^{+}=0.9,0.8,0.7,0.6$ and $\frac{a}{2}=\mu^{-}=\left(1-\mu^{+}\right)$. This gives us optimal quotas $\tilde{\alpha}=\lceil 0.1 n\rceil,\lceil 0.2 n\rceil,\lceil 0.3 n\rceil,\lceil 0.4 n\rceil,\lceil 0.5 n\rceil$ respectively. Using (17), we get the following showing the ratio, $\rho$, of the loss of expected welfare from the optimum as a fraction of the expected welfare attained at the optimal quota $(\tilde{\alpha})$ from using the different (possibly inoptimal) quotas $\alpha$. Table 2 is computed for the case of $n=100$.

Recall that standard computational software (such as "Matlab," "Maple" etc.), using 32-bit processor based computing capability have built in algorithms that are designed to ignore very small (more than 5 significant digit) differences treating them as not being distinguishable ${ }^{19}$. Thus, the zeros in the table

Table 2. Fraction of expected welfare loss from using majority quota.

\begin{tabular}{ccccc}
\hline$\alpha$ & $\rho=\frac{\Delta E W}{\text { OptimalEW }}$ & $\rho=\frac{\Delta E W}{\text { OptimalEW }}$ & $\rho=\frac{\Delta E W}{\text { OptimalEW }}$ & $\rho=\frac{\Delta E W}{\text { OptimalEW }}$ \\
\hline$n=100$ & $\bar{\alpha}=0.1 n$ & $\bar{\alpha}=0.2 n$ & $\bar{\alpha}=0.3 n$ & $\bar{\alpha}=0.4 n$ \\
\hline $0.1 n$ & 0 & 0 & 0 & 0 \\
$0.2 n$ & 0 & 0 & 0 & 0 \\
$0.3 n$ & 0 & 0 & 0 & 0 \\
$0.4 n$ & 0 & 0 & 0 & 0 \\
$0.5 n$ & 0 & 0 & 0 & $6.419 \times 10^{-3}$ \\
$0.6 n$ & 0 & 0 & 0.0087 & 0.35924 \\
$0.7 n$ & 0 & $4.8333 \times 10^{-3}$ & 0.4053 & 0.96121 \\
$0.8 n$ & 0.0007 & 0.41407 & 0.97899 & 0.99997 \\
$0.9 n$ & 0.4025 & 0.99329 & 1.0000 & 1.0000 \\
$n$ & 0.99997 & 1.0000 & 1.0000 & 1.0000 \\
\hline
\end{tabular}

${ }^{19}$ For instance while $\frac{40}{1}$ and $\frac{40}{0.99999}$ are both calulated by the computer as 40.0 even though $\frac{40}{0.9999}$ is shown as 40.004 . 
represent expected welfare levels that differ from the optimal by less than $1000^{\text {th }}$ of a percent. We will say that the welfare levels at these quotas is not measurably different from that at the optimum. Similarly, the ones in the table arise from inoptimal expected welfare levels that are less the $1000^{\text {th }}$ of a percent of the optimal and we will say that virtually 100 per cent of the expected welfare is lost.

Computations similar to the one above for different values of $n$ can be used to make two important observations:

Observation 1: The phenomenon of the majority quota ( $\alpha=0.5 n)$ performing well even when it is inoptimal becomes even more pronounced as the size of $n$ increases.

Observation 2: Counterintuitively, the larger the difference in percentage terms between the optimal quota and the inoptimal quota, the more "difficult" it is to find a measurable difference (in percentage terms) between the optimum expected welfare and the welfare generated by the majority quota. (This can be seen in Table 2 from the row corresponding to $\alpha=0.5 n$ and thus we can see that for the values of the optimum quota $0.1 n, 0.2 n$ and $0.3 n$ a perceptible loss of welfare does not occur if the majority quota is used, while for the optimal quota of $0.4 n$ a perceptible loss of welfare does occur if the majority quota is used.)

To understand what is going on, recall (Remark 5) that for an appropriate selection of the parameters ( $a, b$ and $\theta$ ) the two part uniform distribution in Example 1 is capable of simulating exactly the behavior of expected welfare of any pre-specified distribution of net valuations. Thus, to identify and explain any anomalies or regularities that may arise in any $\alpha$-quota referendum it is sufficient to study the two part uniform distribution of net valuations. This is what we will do and inter alia provide an explanation for observations 1 and 2 above.

Using Proposition 1 and Example 1, we can see that the general form of the expected welfare function for the two part uniform distribution is:

$$
E(W(\alpha))=-\sum_{q=\alpha}^{n} \Delta(q)=\sum_{q=\alpha}^{n}\left[q \frac{b}{2}-(n-q) \frac{a}{2}\right]\left(\begin{array}{l}
n \\
q
\end{array}\right)(1-\theta)^{q}(\theta)^{n-q}
$$

The term inside the summation $(-\Delta(q))$ can be split into two terms:

1) the term $A(q)=\left[q \frac{b}{2}-(n-q) \frac{a}{2}\right]$ and 2) $B(q)=\left(\begin{array}{l}n \\ q\end{array}\right)(1-\theta)^{q}(\theta)^{n-q}$

The term $A(q)$ provides a measure of the deviation from the optimum and $B(q)$ is the standard binomial density function. The expected welfare can thus be viewed as a weighted sum of $A(q) \mathrm{s}$ weighted by the corresponding probability densities of the binomial density function.

A measure of "inefficiency" of the majority based referendum, $\rho$, is given by the ratio of the loss of expected welfare at the inoptimal quota to the expected welfare from using the optimal quota ${ }^{20}$. From Equation (18), for the majority quota, this is given by:

${ }^{20}$ Where $\tilde{\tau} n=\tilde{\alpha}<0.5 n$ is the optimal quota. 


$$
\rho^{m a j}=\frac{\sum_{q=\tilde{\tau} n}^{[0.5 n\rceil-1} A(q) B(q)}{\sum_{q=\tilde{\tau} n}^{[0.5 n\rceil-1} A(q) B(q)+\sum_{q=\lceil 0.5 n\rceil}^{n} A(q) B(q)} .
$$

Here, the denominator is the expected welfare at the optimum and the numerator is the loss of expected welfare from this optimum when the majority quota is used. For this measure $\rho, \rho^{m a j}=0$ represents no measurable loss of expected welfare from using the majority quota as compared to the optimum quota with $\rho^{m a j}=1$ representing a 100 per cent loss ${ }^{21}$.

It is clear from (20) that $\rho^{m a j}$ is determined by three factors:

1) The relative number of integers in the interval $[\tilde{\tau} n,[0.5 n\rceil-1]$ as compared to the number between $[\lceil 0.5 n\rceil, n]$.

2) The relative magnitude of the terms $A(q)$ in the interval $[\tilde{\tau} n,\lceil 0.5 n\rceil-1]$ as compared to those in the interval $[\lceil 0.5 n\rceil, n]$.

3) The relative magnitude of the terms $B(q)$ in the interval $[\tilde{\tau} n,\lceil 0.5 n\rceil-1]$ as compared to its magnitude in the interval $[\lceil 0.5 n\rceil, n]$.

Factors (1) and (2) are determined by the extent of the deviation of the majority quota from the optimal and other things remaining the same, greater the deviation more inefficient these factors would tend to make the majority quota.

Factor (3), on the other hand, is determined by $F(0)=\theta$ and by $n$ which give us the binomial density probabilities weighting each $A(q)$. For large values of $n$, because of large factorial terms involved when computing $\left(\begin{array}{l}n \\ q\end{array}\right)$, this factor can for some values of $n$ and $q$ completely dominate the effect of the other two factors. It is well known that the Binomial distribution is (very) asymmetric for large and small values of $F(0)$ and that greater the deviation of $F(0)$ from 0.5 the more the density skews to one side.

Consider what happens to $\rho^{m a j}$ when $F(0)=\theta<0.5$. Let $(1-\theta)=0.5+\delta$ for $\delta>0$. In this case, Hoeffding's inequality ${ }^{22}$ for the binomial distribution gives us the following bound:

$$
\sum_{q=\tilde{\tau} n}^{\lceil 0.5 n-1\rceil} B(q) \leq \sum_{q=0}^{\lceil 0.5 n\rceil} B(q) \leq \exp \frac{(-2(n(0.5+\delta))-0.5 n)^{2}}{n}=\exp \left(-2 n \delta^{2}\right) .
$$

$A(q)$ (see (19)) can never exceed $\frac{n b}{2}$. Hence,

$$
\sum_{q=\tilde{\tau} n}^{\lceil 0.5 n\rceil-1} A(q) B(q) \leq \sum_{q=\tilde{\tau}_{n}}^{[0.5 n\rceil-1} \frac{n b}{2} B(q)=\frac{n b}{2} \sum_{q=\tilde{\tau} n}^{[0.5 n]-1} B(q) .
$$

Using (21) we get:

\footnotetext{
${ }^{21}$ Note that both the numerator and the denominator of (20) are positive and that the numerator of (20) is necessarily smaller than the denominator (i.e., $1>\rho>0$ ). This is so because from Theorem 1 we know that $\tilde{\tau} n$ is the generically unique optimal quota and the expected welfare function is declining for values of $\alpha>\tilde{\tau} n$. Thus the value of expected welfare at the optimum (the denominator of (20)) and the expected welfare at the majority quota is greater than the expected positive welfare given by (18) when $\alpha=n$.

${ }^{22}$ This well known inequality is used to establish the second inequality in (21). (See [20]).
} 


$$
\sum_{q=\tilde{\tau} n}^{\lceil 0.5 n\rceil-1} A(q) B(q) \leq \frac{n b}{2} \exp \left(-2 n \delta^{2}\right)
$$

For large $n, \frac{n b}{2} \exp \left(-2 n \delta^{2}\right) \rightarrow 0$ and hence from (22), $\rho^{m a j} \rightarrow 0$. Thus, when the optimal $\tau, \tilde{\tau}<0.5$, in large societies there is a negligible loss of expected welfare from using the majority quota ${ }^{23}$.

In general, the larger the value of $|\theta-0.5|$, the faster the convergence to these asymptotic results. This explains Observation 1. Moreover, the fact that the Binomial distribution gets less and less skewed and approaches the normal distribution for large $n$ and the convergence to the asymptotic results slows as $F(0)=\theta \rightarrow 0.5$ explains Observation 2 .

(Analogously, when $(1-\theta)<0.5$, for large $n$, the distribution skews towards and gets concentrated on values of $A(q)$ with $q<0.5$ and the second term in the denominator (20) becomes negligible for large $n$ and virtually a 100 per cent loss of expected welfare $(\rho \rightarrow 1)$ is possible from the inoptimal use of the majority quota!)

More generally, for any two part uniform distribution of net valuation, consider three possible scenarios $\mathrm{A}, \mathrm{B}$ and $\mathrm{C}$ of underprovision under the majority quota:

A. If $F(0)$ is very small then, for large $n$, the probability densities are concentrated on values of $q>0.5 n$. Virtually no loss in expected welfare will occur from using the majority quota because of the vanishingly small values of $B(q)$ between the optimal and majority quota.

B. If $F(0) \approx 0.5$ then, for large $n$, the binomial approaches the normal distribution and the weighting of the terms $A(q)$ between $q=\tilde{\alpha}$ and $q=\lceil 0.5 n\rceil$ is significant particularly for the terms near $0.5 n$. Therefore, there will be an easily observed loss in expected welfare from using the majority quota rather than the optimum quota ${ }^{24}$.

C. If $F(0)$ is very large then, for large $n$, the binomial density weight on the terms $A(q)$ for $q>0.5$ is negligible and an almost complete loss in expected welfare from the inoptimal use of the majority quota based referendum is possible ${ }^{25,26}$.

Thus, recalling the fact that small optimal proportions occur for small values of the ratio of positive to negative means we conjecture that the following is true:

Conjecture: In large societies, if the relative intensity of the support among

\footnotetext{
${ }^{23}$ In this argument we use the fact that $\rho>0$.

${ }^{24}$ For instance, if $n=100, \tilde{\alpha}=25 ;(1-F(0))=0.5$ then the percentage loss of welfare as compared to that at the optimum quota will be approximately $38.062 \%$.

${ }^{25}$ For instance, if $n=100, \tilde{\alpha}=25 ;(1-F(0))=0.1$ then the percentage loss of welfare as compared to that at the optimum quota will be almost $100 \%$.

${ }^{26}$ In equation (20), what may happen as $n \rightarrow \infty$, is that the lower tail $\sum_{q=[0.5 n]}^{n} A(q) B(q) \rightarrow 0$ faster than $\sum_{q=\bar{z} n}^{[0.5 n]-1} A(q) B(q) \rightarrow 0$ and hence it is possible for $\frac{\sum_{q=[0.5 n\rceil}^{n} A(q) B(q) \rightarrow 0}{\sum_{q=\bar{z} n}^{[0.5 n]-1} A(q) B(q)} \rightarrow 0$ and for a $100 \%$ loss of welfare to occur.
} 
supporters as compared to the intensity of the opposition among opponents $\left(\frac{\mu^{+}}{\mu^{-}}\right)$is positively associated with a large probability of a positive net valuation $(1-F(0))$, then the use of the majority quota will lead to insignificant losses in percentage terms of expected welfare as compared to the expected welfare that is generated by the optimum quota.

The intuition underlying the above conjecture is as follows: expected welfare depends both on the intensity and the extent of the support. While the majority quota it is a good at identifying whether or not the extent of the support is on the average positive it may or may not be a good signal of the relative intensity of support. When these two factors, extent and intensity, are positively associated it performs well. Analogously, the loss in expected welfare from using the majority quota (when the majority quota is not optimal) will be large and the use of the majority quota in the presence of intense minority opposition is likely to give poor results.

\section{Conclusion}

Our analysis of quota based referendum rules for public goods yielded a closed form solution for the optimal quota in terms of the parameters of the model: the positive and negative means and the size of the population. The solution is almost always unique and is independent of any other "higher order moments" of the distribution of net valuations. Deviations on either side of this (unique) optimal quota results in the loss of expected welfare. This welfare loss increases the further the actual quota deviates on any one side from this optimal quota. Our formula for the optimum quota implies that the optimum tends to be positively related to the negative mean of the net valuation and it tends to depend negatively on the positive mean of the net valuation. If positive and negative mean net valuations are equal, the majority quota is optimum. The per capita cost can change the optimal quota by changing the positive and negative means. In general, the impact of this effect is ambiguous. The behavior of the optimal quota when the size of the population increases depends on the cost structure. Under reasonable assumptions about the cost structure, the optima quota grows asymptotically at the rate of growth of the population. Deviations from the optimum may or may not make a significant difference to expected welfare. In large societies, if the majority quota is used instead of the optimum quota and the relative intensity of the support among supporters as compared to the intensity of the opposition among opponents, is a good indicator of the "number of supporters" (i.e., of the probability of an average individual of having a positive net valuation) then the majority quota will provide satisfactory results.

\section{Conflicts of Interest}

The authors declare no conflicts of interest regarding the publication of this paper. 


\section{References}

[1] Kuzmics, C. and Steg, J. (2017). On Public Good Provision Mechanisms with Dominant Strategies and Balanced Budget. Journal of Economic Theory, 170, 56-69. https://doi.org/10.1016/j.jet.2017.04.006

[2] Clarke, E. (1971) Multipart Pricing of Public Goods. Public Choice, 11, 17-33. https://doi.org/10.1007/BF01726210

[3] Groves, T. (1973) Incentives in Teams. Econometrica, 41, 617-663. https://doi.org/10.2307/1914085

[4] Deb, R. and Ohseto, S. (1999) Strategy-Proof and Individually Rational Social Choice Functions for Public Good Economies: A Note. Economic Theory, 14, 685-689. https://doi.org/10.1007/s001990050348

[5] Deb, R. and Razzolini, L. (1999) Auction Like Mechanisms for Pricing Excludable Public Goods. Journal of Economic Theory, 88, 340-368. https://doi.org/10.1006/jeth.1999.2603

[6] Ohseto, S. (2003) Augmented Serial Rules for an Excludable Public Good. Economic Theory, 26, 589-606. https://doi.org/10.1007/s00199-004-0536-y

[7] Hashimoto, K. and Saitoh, H. (2016) Strategy-Proof Rules for an Excludable Public Good. Social Choice and Welfare, 46, 749-766. https://doi.org/10.1007/s00355-015-0933-0

[8] Groves, T. and Ledyard, J. (1977) Optimal Allocation of Public Goods: A Solution to the Free Rider Problem. Econometrica, 45, 783-809. https://doi.org/10.2307/1912672

[9] Ledyard, J. and Palfrey, T. (2007) A General Characterization of Interim Efficient Mechanisms for Independent Linear Environments. Journal of Economic Theory, 132, 441-466. https://doi.org/10.1016/j.jet.2005.12.006

[10] Masso, J., Nicolo A., Sen, A., Sharma, T. and Ulku, L. (2015) On Cost Sharing in the Provision of a Binary and Excludable Public Good. Journal of Economic Theory, 155, 30-49. https://doi.org/10.1016/j.jet.2014.11.003

[11] Bierbrauer, F. and Hellwig, M. (2016) Robustly Coalition-Proof Incentive Mechanisms for Public Good Provision are Voting Mechanisms and Vice Versa. Review of Economic Studies, 83, 1440-1464. https://doi.org/10.1093/restud/rdw015

[12] Deb, R. and Seo, T.K. (2010) Strategy-Proofness and Public Good Provision Using Referenda Based on Unequal Cost Sharing. International Journal of Game Theory, 39, 222-231. https://doi.org/10.1007/s00182-009-0192-3

[13] Ledyard, J. and Palfrey, T. (2002) The Approximation of Efficient Public Good Mechanisms by Simple Voting Schemes. Journal of Public Economics, 83, 153-171. https://doi.org/10.1016/S0047-2727(00)00161-4

[14] Schmitz, P. and Troger, T. (2012) The Sub-Optimality of the Majority Rule. Games and Economic Behavior, 74, 651-655. https://doi.org/10.1016/j.geb.2011.08.002

[15] Matsusaka, J. (2005) The Eclipse of Legislatures: Direct Democracy in the 21st Century. Public Choice, 124, 157-177. https://doi.org/10.1007/s11127-005-4760-1

[16] Ballotwatch (2010-2017) Initiative and Referendum Institute, University of Southern California. http://www.iandrinstitute.org

[17] The Tom Warne Report (2014) http://www.tomwarne.com/news/2014/11/election-update-2014-voters-weigh-in-on -transportation-issues/

[18] Ledyard, J. (2006) Voting and Efficient Public Good Mechanisms. In: Weingast, B. 
and Wittman, D., Eds., The Oxford Handbook of Political Economy, Oxford University Press, 479-501.

[19] Barbera, S. and Jackson, M. (2006) On Weights of Nations: Assigning Voting Weights in Heterogenous Unions. Journal of Political Economy, 114, 317-339. https://doi.org/10.1086/501172

[20] Hoeffding, W. (1963) Probability Inequalities for Large Sums of Bounded Random Variables. Journal of the American Statistical Association, 58, 13-30. https://doi.org/10.1080/01621459.1963.10500830 